\title{
Effect of spin-orbit interactions on the 0.7 anomaly in quantum point contacts
}

\author{
Olga Goulko, ${ }^{1,2}$ Florian Bauer, ${ }^{1}$ Jan Heyder, ${ }^{1}$ and Jan von Delft ${ }^{1}$ \\ ${ }^{1}$ Physics Department, Arnold Sommerfeld Center for Theoretical Physics, and Center for NanoScience, \\ Ludwig-Maximilians-Universität, Theresienstraße 37, 80333 Munich, Germany \\ ${ }^{2}$ Present address: Department of Physics, University of Massachusetts, Amherst, MA 01003, USA
}

\begin{abstract}
We study how the conductance of a quantum point contact is affected by spin-orbit interactions, for systems at zero temperature both with and without electron-electron interactions. In the presence of spin-orbit coupling, tuning the strength and direction of an external magnetic field can change the dispersion relation and hence the local density of states in the point contact region. This modifies the effect of electron-electron interactions, implying striking changes in the shape of the 0.7-anomaly and introducing additional distinctive features in the first conductance step.
\end{abstract}

PACS numbers: 71.70.Ej, 73.40.-c

Spin-orbit interactions (SOI) play an important role in a variety of fields within mesoscopic physics, such as spintronics and topological quantum systems. In this Letter we study the effects of SOI on the conductance of a quantum point contact (QPC), a one-dimensional constriction between two reservoirs $[1,2]$. The linear conductance $G$ of a QPC is quantized in multiples of $G_{\mathrm{Q}}=2 e^{2} / h$, showing the famous staircase as a function of gate voltage. In addition, at the onset of the first plateau, measured curves show a shoulderlike structure near $0.7 G_{\mathrm{Q}}$ [3]. In this regime QPCs exhibit anomalous behavior in the electrical and thermal conductance, noise, and thermopower [3-11]. The microscopic origin of this 0.7 -anomaly has been the subject of a long debate [12-18]. It has recently been attributed to a strong enhancement of the effects of electron-electron interactions (EEI) by a smeared van Hove singularity in the local density of states (LDOS) at the bottom of the lowest QPC subband [15, 18]. While this explains the 0.7 -anomaly without evoking SOI, the presence of SOI can change the dispersion relation and hence the LDOS, thus strongly affecting the shape of the 0.7 -anomaly. Previous studies of SOI in QPCs exist [1923], but not with the present emphasis on their interplay with the QPC barrier shape and EEI, which are crucial for understanding the effect of SOI on the 0.7-anomaly.

Setup. We consider a heterostructure forming a twodimensional electron system (2DES) in the $x y$-plane. Gate voltages are used to define a smooth, symmetric potential which splits the 2DES into two leads, connected by a short, one-dimensional channel along the $x$-axis: the QPC $[1,2]$. The transition between the leads and the QPC is adiabatic. We also assume the confining potential in the transverse direction to be so steep that the subband spacing is much larger than all other energy scales relevant for transport, in particular those related to the magnetic field and SOI, and consider only transport in the first subband, corresponding to the lowest transverse mode. This can be described by a one-dimensional model with a smooth potential barrier and local EEI [18]. The magnetic field $\boldsymbol{B}$ is assumed to be in the $x y$-plane, acting as a pure Zeeman field, without orbital effects.

A moving electron in an electric field can experience an effective magnetic field $\boldsymbol{B}_{\text {SOI }}$ proportional to its momen- tum $\hbar k$. Depending on the origin of the electric field one distinguishes between Rashba and Dresselhaus terms, the former resulting from the gradient of the external potential, and the latter from the asymmetry of the ionic lattice [24]. To be able to rotate $\boldsymbol{B}$ through any angle $\varphi$ w.r.t. $\boldsymbol{B}_{\mathrm{SOI}}$ we require that $\boldsymbol{B}_{\mathrm{SOI}}$ also lies in the $x y$ plane. W.l.o.g. (see Supplement) we choose the $y$-axis to be parallel to $\boldsymbol{B}_{\mathrm{SOI}}$, such that the SOI contribution to the Hamiltonian is $-\alpha \sigma_{y} k$, where $\alpha$ characterizes the strength of the (Rashba) SOI and $\sigma_{y}$ is a Pauli matrix [25]. We only consider the leading SOI contribution proportional to $k$ and choose the spin quantization direction along $\boldsymbol{B}$.

Without SOI, the dispersion relation $\hbar^{2} k^{2} / 2 m$ of a homogeneous one-dimensional model with effective mass $m$ splits in the presence of a Zeeman field into two identical branches offset in energy by $\pm B / 2$. On the other hand, without a Zeeman field, the momentum-dependent SOI splits the dispersion in $k$-direction and also yields a negative spin-independent energy offset of magnitude $\Delta E_{\mathrm{SOI}}=\alpha^{2} m / 2 \hbar^{2}$. In the following we shift the energy origin by $-\Delta E_{\mathrm{SOI}}$ and quote all energies w.r.t. the new origin. If both $\boldsymbol{B}$ and $\boldsymbol{B}_{\mathrm{SOI}}$ are non-zero, their interplay depends on $\varphi$, as illustrated in Fig. 1(a1-a3). In (a1), where the fields are parallel $(\varphi=0)$, the energy offsets simply add, while for nonparallel fields a spin mixing occurs, resulting in an avoided crossing [26]. For orthogonal fields $(\varphi=\pi / 2)$, the lower dispersion branch exhibits either one broader minimum at $k=0$ if $B \geq 4 E_{\mathrm{SOI}}$, or two minima at finite $k$ and a maximum at $k=0$ otherwise. The latter case is shown in Fig. 1(a2-a3).

Model. For the lowest subband we model the QPC by a symmetric potential barrier which is quadratic around its maximum,

$$
V(x) \simeq V_{\mathrm{g}}+\mu-C_{\mathrm{b}} x^{2} / 2,
$$

and vanishes smoothly at the boundary of the QPC. The barrier height $V_{\mathrm{g}}$, measured w.r.t. the chemical potential $\mu$, mimics the role of the gate-voltage. If $V_{\mathrm{g}}$ is swept downwards through zero, the conductance $g=G / G_{\mathrm{Q}}$ increases from 0 to 1 . For $B=0$ this occurs in a single step whose width is given by the energy scale $\Omega_{x}=\sqrt{C_{\mathrm{b}} C_{\mathrm{d}}}$, 


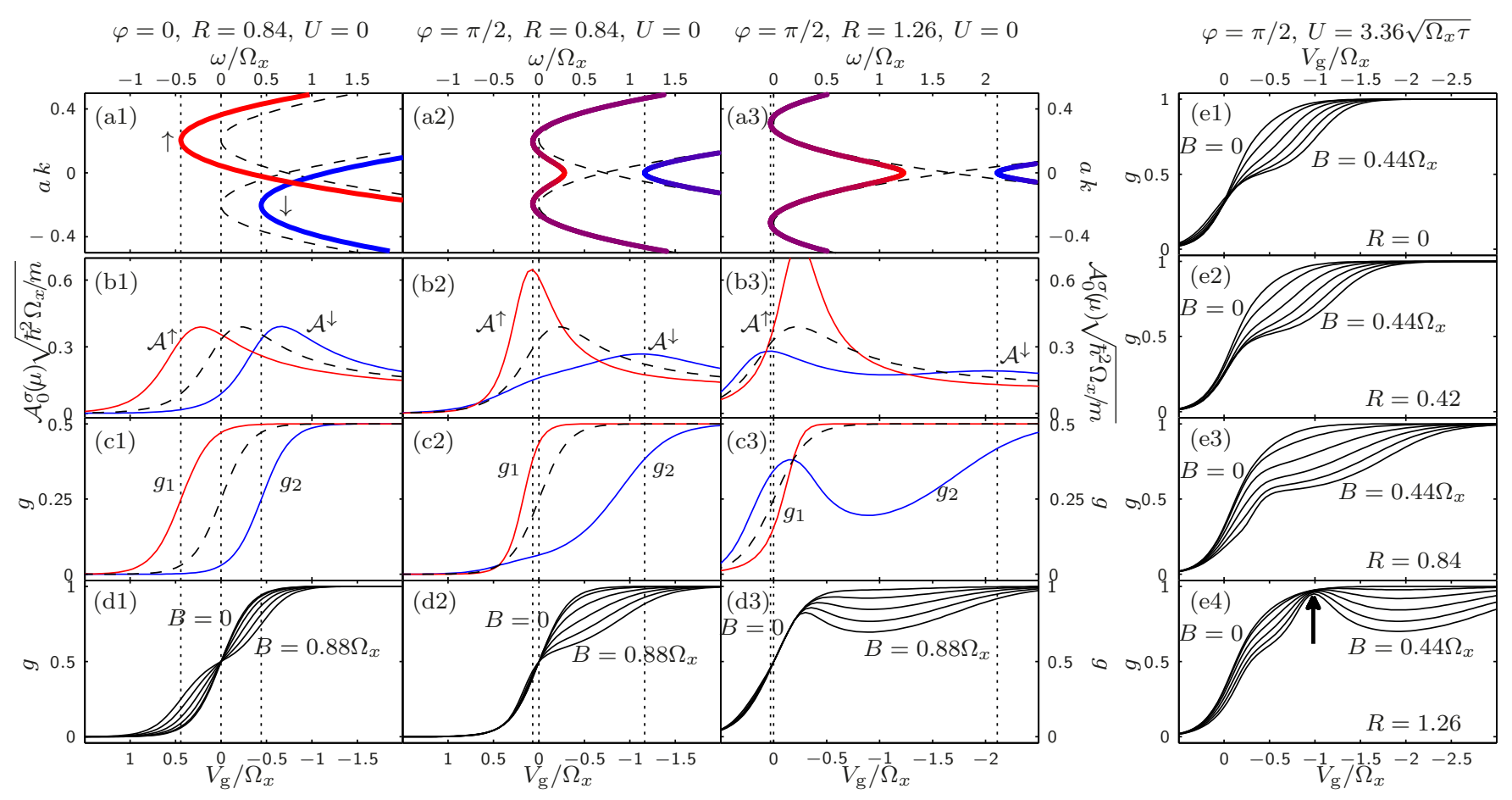

FIG. 1. Effect of SOI on the model without EEI, left columns (a1-d3), and with EEI, right column (e1-e4). The left columns (a1-d1), (a2-d2) and (a3-d3) represent different combinations of SOI strength $R$ and angle $\varphi$ between $\boldsymbol{B}$ and $\boldsymbol{B}_{\text {SOI }}$. They highlight the correspondence between the dispersion relation $\omega(k)$ in a homogeneous system (a1-a3), the LDOS for fixed $\omega=\mu$ as function of $V_{\mathrm{g}}$ on the central site of a QPC with potential barrier (b1-b3), the conductances of the two QPC transmission channels (c1-c3), and the total conductance of the QPC for several equally-spaced magnetic field values between $B=0$ and $B=0.88 \Omega_{x}(\mathrm{~d} 1-\mathrm{d} 3)$. In (a1-c3) the magnetic field is fixed at $B=0.88 \Omega_{x}$, with dashed lines showing the case $B=0$ for comparison. The line colors in (a1-a3) quantify the contribution of each spin state (red= $\uparrow$, blue $=\downarrow$ ) in the dispersion branches, to illustrate the spin mixing at $\varphi \neq 0$. The right column (e1-e4) shows the total conductance for $U>0$, with $\varphi=\pi / 2$ and several combinations of $R$ and $B$ (the latter were chosen smaller than in (d1-d3), since EEI enhance the $g$-factor [18]).

which is set by the fixed curvature of the barrier, $C_{\mathrm{b}}$, and the curvature of the bulk dispersion at its minimum, $C_{\mathrm{d}}$ [27]. For $\varphi=0, C_{\mathrm{d}}=\hbar^{2} / m$.

For numerical purposes we discretize real space and obtain an infinite tight-binding chain with spacing $a$, taking $\boldsymbol{B}$ and $\alpha$ constant throughout the chain. The noninteracting Hamiltonian is

$$
\begin{aligned}
H_{0}= & \sum_{j, \sigma, \sigma^{\prime}} d_{j \sigma}^{\dagger}\left[\left(V_{j}+2 \tau\right) \delta_{\sigma \sigma^{\prime}}-\frac{1}{2}(\boldsymbol{\sigma} \cdot \boldsymbol{B})_{\sigma \sigma^{\prime}}\right] d_{j \sigma^{\prime}} \\
& +\sum_{j, \sigma, \sigma^{\prime}}\left[d_{j+1 \sigma}^{\dagger}\left(-\tau_{0} \delta_{\sigma \sigma^{\prime}}+\frac{i \alpha}{2}\left(\sigma_{y}\right)_{\sigma \sigma^{\prime}}\right) d_{j \sigma^{\prime}}+\text { h.c. }\right]
\end{aligned}
$$

where $d_{j \sigma}$ annihilates an electron with spin $\sigma \in\{\uparrow, \downarrow\} \equiv$ $\{+,-\}$ at site $j$. The effective mass of the charge carrier is $m=\hbar^{2} / 2 \tau a^{2}$ with $\tau=\sqrt{\tau_{0}^{2}+\alpha^{2}}$ [28]. We keep $\tau$ fixed when varying $\alpha$. The QPC barrier potential $V_{j}=V(j a)$ (and later EEI) are nonzero only in a region of length $L=2 N a$ centered around $j=0$, representing the QPC. All results shown are for $N=50$. We use the smooth function $V(x)=\left(V_{\mathrm{g}}+\mu\right) \exp \left[-(2 x / L)^{2} /\left(1-(2 x / L)^{2}\right)\right]$ for the potential, with $\mu=2 \tau$. Sites $j<-N$ and $j>N$ represent two leads with bandwidth $4 \tau$. The strength of
SOI in a QPC is determined by the dimensionless parameter

$$
R=\sqrt{\frac{\Delta E_{\mathrm{SOI}}}{\Omega_{x}}}=\frac{\alpha}{\hbar} \sqrt{\frac{m}{2 \Omega_{x}}} .
$$

SOI strengths of up to $\alpha \simeq 10^{-11} \mathrm{eVm}$ have been reported in the literature $[19,26,29,30]$. Typical values of $\Omega_{x} \simeq 1 \mathrm{meV}$ and $m \simeq 0.05 m_{e}$ for InGaAs yield $R \simeq 0.2$. A stronger spin-orbit effect due to an enhancement of the anisotropic Lande $g$-factor is reported in [31]. Hole quantum wires have been used to observe the spin-orbit gap [26] and the anisotropic Zeeman splitting [32]. For hole QPCs, the larger effective hole mass and the resulting smaller $\Omega_{x}$ imply larger values of $R$. Here we consider both small and large $R$, where $R \lesssim 0.4$ is a realistic scale for electron systems and $R \gtrsim 1$ is accessible using hole systems [33], for QPCs with small barrier curvature $C_{\mathrm{b}}$ and hence small $\Omega_{x}$.

System without EEI. Many insights on the interplay between SOI and geometry can already be gained from the model without EEI, as shown in the left part (a1d3) of Fig. 1. We discuss exact results for two physical quantities, which we also relate to the bulk dispersion relation: the linear conductance $g$ and the LDOS 
$\mathcal{A}_{j}^{\sigma}(\omega)=-\operatorname{Im} \mathcal{G}_{j j}^{\sigma \sigma}(\omega) / \pi a$, where $\mathcal{G}_{j j^{\prime}}^{\sigma \sigma^{\prime}}$ is the retarded propagator from site $j^{\prime}$ with spin $\sigma^{\prime}$ to site $j$ with spin $\sigma$. Due to SOI, spin is not conserved for $\varphi \neq 0$ and hence $\mathcal{G}_{j j}^{\sigma \sigma^{\prime}}$ is not spin-diagonal. However at $j=0$ its off-diagonal elements turn out to be negligible compared to the diagonal ones. Thus it is meaningful to analyze the LDOS at $j=0$ for given $\sigma$. The linear conductance at zero temperature can be calculated via $g=g_{1}+g_{2} \propto \operatorname{Tr}\left(t^{\dagger} t\right)$ [34], where $t^{\sigma \sigma^{\prime}}=\mathcal{G}_{-N, N}^{\sigma \sigma^{\prime}}(\mu)$ is the transmission matrix of the QPC and $\operatorname{Tr}\left(t^{\dagger} t\right)$ equals the sum of the eigenvalues of $t^{\dagger} t$. The spin structure of $t$ depends on $N$, but the eigenvalues of $t^{\dagger} t$, which yield the conductances $g_{1}$ and $g_{2}$ of the two transmission channels, do not.

For $\varphi=0$ (Fig. 1, left column) spin is conserved and SOI have no influence on the LDOS and the conductance. This case is analogous to the one discussed in [18]. The bulk (i.e. $V(x)=0$ ) LDOS,

$$
\left.\mathcal{A}_{\text {bulk }}^{\sigma}(\omega) \propto \frac{\partial k}{\partial \omega}\right|_{\sigma}=\sqrt{\frac{m}{2 \hbar^{2}(\omega+\sigma B / 2)}},
$$

has a van Hove singularity, diverging at the minimum $\omega=-\sigma B / 2$ of the corresponding dispersion branch, where the electron velocity vanishes. In the $\mathrm{QPC}$, the $x$ dependent LDOS is shifted in energy by the barrier potential $V(x)$. Since the barrier breaks translational invariance, the van Hove singularity is smeared out on a scale set by $\Omega_{x}$ [15], forming a ridgelike structure, called van Hove-ridge in [18]. The LDOS height becomes finite, of order $\mathcal{O}\left(\sqrt{m /\left(\hbar^{2} \Omega_{x}\right)}\right)$, determined by $\Omega_{x}$ and the curvature $\hbar^{2} / m$ of the bulk dispersion. At a given position $x$, the LDOS maximum occurs at an energy which is $\mathcal{O}\left(\Omega_{x}\right)$ larger than the corresponding potential energy $V(x)-\sigma B / 2$. Here and henceforth we quote the LDOS as a function of $V_{\mathrm{g}}$ at fixed $\omega=\mu$. Figure 1(b1) shows it at the central site $j=0$; the spatially resolved LDOS is shown in Fig. 1 of the Supplement. The LDOS has the same shape for both spins. Its structure is clearly inherited from that of the dispersion in (a1), with peak energies aligned with the dispersion minima up to the shift of $\mathcal{O}\left(\Omega_{x}\right)$. Similarly, the conductances $g_{1}\left(V_{\mathrm{g}}\right)$ and $g_{2}\left(V_{\mathrm{g}}\right)$ of the two channels in (c1) show steps of the same shape with widths $\propto \Omega_{x}$ [27], split by $B$ and aligned with the dispersion minima. This causes the total conductance $g\left(V_{\mathrm{g}}\right)$ in $(\mathrm{d} 1)$ to split symmetrically into a double step with increasing field, just as for a QPC without SOI.

Next consider the case $\varphi=\pi / 2$ shown in Fig. 1(a2-d3). Spin mixing leads to an avoided crossing with spin gap $\propto B$, which splits the dispersion into an upper branch with a narrow minimum and a lower branch with two minima and one maximum (for $B<4 E_{\mathrm{SOI}}$ ). Note that bulk LDOS structures separated in energy by less than $\Omega_{x}$ are not resolved within the QPC. In the following we give an intuitive explanation of how the dispersion minima relate to the properties of the LDOS peaks and the conductance steps. The curvatures of the lower and upper dispersion branches are, respectively, smaller or larger than in (a1), $C_{\mathrm{d} 1}<C_{\mathrm{d}}<C_{\mathrm{d} 2}$ (loosely speaking, $C_{\mathrm{d} 1}$ is the effective curvature obtained by smearing the double dispersion minimum by $\Omega_{x}$, yielding a single minimum). Because the barrier curvature $C_{\mathrm{b}}$ is fixed, this results in two modified energy scales $\Omega_{x i}=\sqrt{C_{\mathrm{b}} C_{\mathrm{d} i}}$, with $\Omega_{x 1}<\Omega_{x}<\Omega_{x 2}$, which determine the LDOS peak heights and widths, as well as the conductance step widths. Consequently, in (b2) the LDOS peak for $\mathcal{A}_{0}^{\downarrow}$ is lower and wider than for $\mathcal{A}_{0}^{\uparrow}$. Likewise, in (c2) the conductance step for $g_{2}\left(V_{\mathrm{g}}\right)$ is wider than for $g_{1}\left(V_{\mathrm{g}}\right)$, causing $g\left(V_{\mathrm{g}}\right)$ in (d2) to show a striking asymmetry for its $B$-induced evolution from a single to a double step. This asymmetry is reminiscent of but unrelated to that known for the 0.7-anomaly - the latter is driven by EEI, as discussed below - but should be observable in higher conductance steps, where EEI are weaker.

For $R \gtrsim 1$ more structures emerge, see Fig. 1(a3-b3). Spin-mixing produces an additional "emergent" peak in $\mathcal{A}_{0}^{\downarrow}(\mathrm{b} 3)$ and an additional step in $g_{2}\left(V_{\mathrm{g}}\right)(\mathrm{c} 3)$ near $V_{\mathrm{g}} \simeq 0$. Between the two steps, the transmission $g_{2}\left(V_{\mathrm{g}}\right)$ has a minimum, corresponding to the spin gap, and the total conductance $g\left(V_{\mathrm{g}}\right)$ in (d3) likewise develops a spin gap minimum with increasing $B$. These features can be understood by looking at the spin composition of the two bulk dispersion branches, depicted quantitatively through the colors in Fig. 1(a1-a3). At $k=0$ the SOI field is zero and we have pure spin-states w.r.t. the chosen quantization. At larger $|k|$ the SOI field increases, leading to spin-mixing. In fact in the limit $k \rightarrow \infty$ we find a fully mixed state with equal up/down contributions. Since the upper branch minimum at $k=0$ is in a pure spin-down state it corresponds to a peak only in $\mathcal{A}^{\downarrow}$. But the minima of the lower branch are shifted away from $k=0$ and have a spin down share besides the dominant spin up contribution. This causes the emergent peak in $\mathcal{A}^{\downarrow}$ at low frequencies, whose height increases with $R$, due to the stronger spin-mixing.

Interacting system. We now include EEI via $H_{\mathrm{int}}=\sum_{j} U_{j} d_{j \uparrow}^{\dagger} d_{j \uparrow} d_{j \downarrow}^{\dagger} d_{j \downarrow}$. The on-site interaction $U_{j}=U(j a)$ is switched on smoothly over the QPC according to $U(x)=U \exp \left(-(2 x / L)^{6} /\left(1-(2 x / L)^{2}\right)\right)$. We set $U_{j}=0$ for $|j|>N$, because outside the QPC region transverse confinement is weak or absent, and screening strong $[18,35]$. We calculate the conductance at zero temperature with the functional Renormalization Group technique in the one-particle irreducible version $[28,36$ 39] using the coupled ladder approximation, which was presented in [35] for a model without SOI. Generalizations necessary in the presence of SOI are described in the Supplement.

The $B$-dependence of the conductance for $\varphi=\pi / 2$ and different $R$ in the presence of EEI is shown in the right column (e1-e4) of Fig. 1 and the corresponding transconductance $\mathrm{d} g / \mathrm{d} V_{\mathrm{g}}$ in Fig. 2(b-f). The case $R=0$, see Figs. 1(e1) and 2(a-c), which is equivalent to $\varphi=0$, has been discussed in $[18,35]$ : once a finite magnetic field breaks the spin degeneracy a surplus of spin-up electrons 

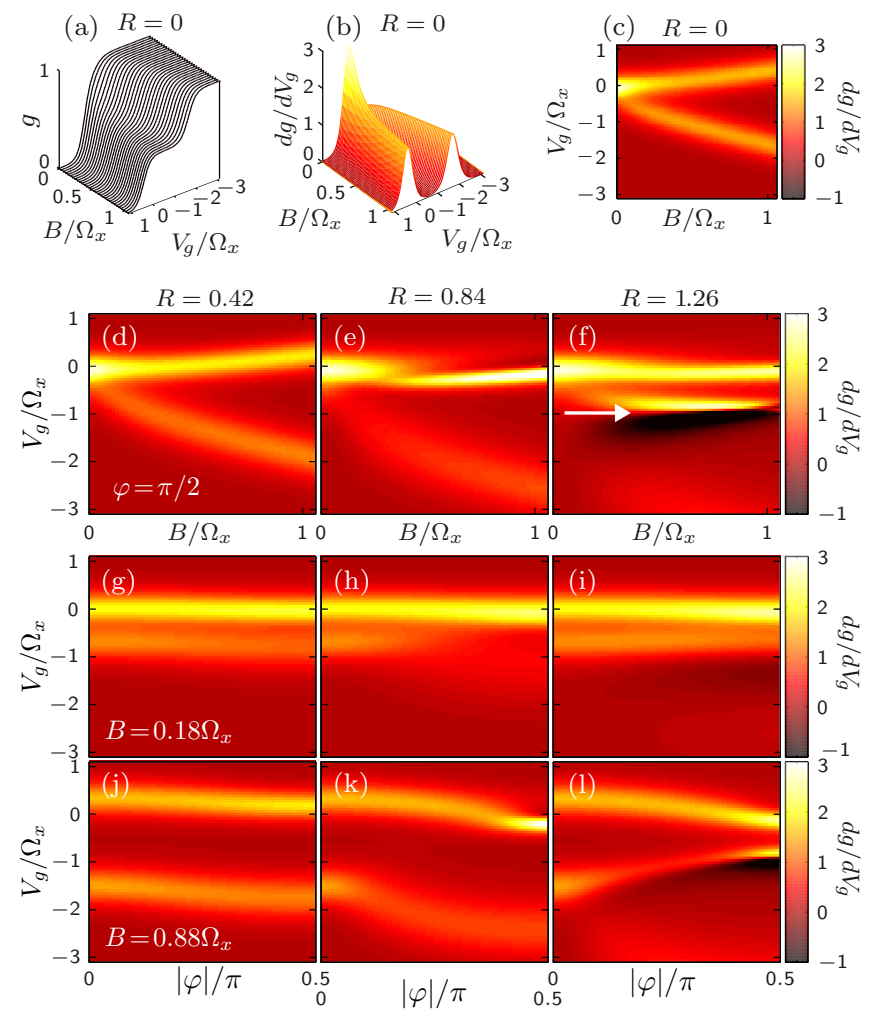

FIG. 2. fRG results for the conductance $g$ and transconductance $d g / d V_{\mathrm{g}}$, for $U=3.36 \sqrt{\Omega_{x} \tau}$ at zero temperature. Top row: $3 \mathrm{~d}$ or colorscale plots of the conductance (a) and the transconductance (b,c) as functions of $V_{\mathrm{g}}$ and $B$, for $R=0$. Three bottom rows (d-l): Colorscale plots of the transconductance for three choices of $R$ (three columns), plotted as a function of $V_{\mathrm{g}}$ and either $B$ for fixed $\varphi=\pi / 2$, (second row), or of $\varphi$ for fixed $B=0.18 \Omega_{x}$ (third row) and $B=0.88 \Omega_{x}$ (fourth row).

develops in the QPC, so that spin-down electrons experience both a Zeeman and a Coulomb energy cost. This Stoner-type effect depends on the LDOS at $\mu$ and hence is strongest when the apex of the van Hove ridge touches the chemical potential, i.e. when $V_{\mathrm{g}}$ is within $\simeq 0.5 \Omega_{x}$ below 0 [18]. This causes an asymmetry w.r.t. $V_{\mathrm{g}}=0$ in the $B$-induced evolution of $g\left(V_{\mathrm{g}}\right)$ from a single to a double step in Fig. 1(e1), in contrast to the case without EEI in Fig. 1(d1). This asymmetry is characteristic of the 0.7 -anomaly. The corresponding transconductance in Fig. 2(b-c) shows a double peak whose spacing increases roughly linearly with $B$ (with an EEI-enhanced g-factor), as seen in numerous experiments $[3,10,18]$.

The Stoner-type Coulomb enhancement of a fieldinduced population imbalance is amplified when $R \neq 0$, as shown in Figs. 1(e2-e4) and 2(d-f), because of the height imbalance for the spin-up and spin-down LDOS peaks caused by SOI. Correspondingly, with increasing $R$ the double-step structure in the conductance becomes more pronounced, the second substep becoming much broader than the first, see Figs. 1(e2-e3), and the transconductance in Fig. 2(d-e) shows a weakening of the lower- $V_{\mathrm{g}}$ peak with increasing $R$. This reflects the increasing curvature $C_{\mathrm{d} 2}$ of the upper dispersion branch (and hence larger step width $\Omega_{x 2}$ ). For $R \gtrsim 1$, additional features, inherited from the noninteracting case, emerge for $g\left(V_{\mathrm{g}}\right)$ in Fig. 1(e4): a local maximum (marked by an arrow), followed by a spin gap minimum at lower $V_{\mathrm{g}}$. For the transconductance, Fig. 2(f), these features show up as a strong secondary peak around $V_{\mathrm{g}} / \Omega_{x} \simeq-1$ (marked by an arrow), followed by a region of negative transconductance (black). EEI also induce a secondary 0.7-type double-step feature in $g\left(V_{\mathrm{g}}\right)$ for $V_{\mathrm{g}} / \Omega_{x}$ between 0 and -1 , Fig. 1(e4), which is similar to, but narrower than that for $R=0$. It originates from the main LDOS peak in $\mathcal{A}_{0}^{\uparrow}$ and the emergent peak in $\mathcal{A}_{0}^{\downarrow}$. Unlike the regular $\mathcal{A}_{0}^{\downarrow}$ peak aligned with the upper dispersion branch, whose $V_{\mathrm{g}}$-position is governed by the magnetic field, the emergent $\mathcal{A}_{0}^{\downarrow}$ peak occurs, due to strong spin-mixing, at nearly $B$-independent energy close to the $\mathcal{A}_{0}^{\uparrow}$ peak. As a result, the two transconductance maxima in Fig. 2(f) remain parallel with increasing $B$, in strong contrast to the situation for $R<1$ in Fig. 2(c-e).

Figures 2(g-l) show, for two fixed values of $B$, how the transconductance evolves as $|\varphi|$ is increased from 0 to $\pi / 2$, thus switching on the effects of SOI. The decrease in peak spacing with increasing $|\varphi|$ in Fig. 2(l) strikingly reflects the increasing importance of spin mixing. The strong angle-dependence predicted here is a promising candidate for an experimental test of our theory [40].

At small nonzero temperature, inelastic scattering causes a Fermi-liquid-type reduction of the conductance, $g\left(T, V_{\mathrm{g}}\right) / g\left(0, V_{\mathrm{g}}\right)=1-\left(T / T_{*}\right)^{2}$ for $T \ll T_{*}$, with a $V_{\mathrm{g}^{-}}$ dependent low-energy scale $T_{*}\left(V_{g}\right)$. We expect its magnitude to be similar to the case without SOI, typically $\simeq 1 \mathrm{~K}$ [18]. Thus, for $T \lesssim 0.1 \mathrm{~K}$, the $T$-dependence should be very weak and the $\widetilde{T}=0$ predictions applicable.

In summary, we have shown that in the presence of SOI, the changes in the dispersion induced by the interplay of $\boldsymbol{B}$ and $\boldsymbol{B}_{\mathrm{SOI}}$ can strongly affect the shape of the 0.7-anomaly. In the absence of EEI, SOI cause an anisotropic response of the spin splitting to the applied in-plane magnetic field. With EEI, the 0.7 -anomaly also develops an anisotropic response to magnetic field, and if SOI are strong, the conductance develops additional features due to the interplay of EEI and SOI: for $\varphi=\pi / 2$ these include a field-induced double step in the conductance that does not split linearly with $B$, followed by a spin gap minimum. The dependence of the conductance on the angle between $\boldsymbol{B}$ and $\boldsymbol{B}_{\mathrm{SOI}}$ is already apparent for $R \simeq 0.4$, which is accessible in experiments with electron QPCs. Hole QPCs with $R \gtrsim 1$ would allow access to regimes with strong SOI.

An experimental verification of our predictions would highlight the influence of LDOS features on the conductance and thus lend further support to the van Hove scenario of Ref. [18] as microscopic explanation for the 0.7 -anomaly. More generally, our work lays out a conceptual framework for analyzing the interplay of SOI, 
EEI and barrier shape in quasi-1D geometries: examine how SOI and barrier shape modify the (bare) LDOS near $\mu$ - whenever the LDOS is large, EEI effects are strong. We expect this to be relevant for the more complicated hybrid superconductor-semiconductor junctions currently studied by seekers of Majorana fermions [4143]. A proper analysis of such systems would require a generalization of our approach to include superconduct- ing effects.

We thank S. Fischer, A. Hamilton, K. Hudson, S. Ludwig, C. Marcus, A. Micolich and A. Srinivasan for interesting and useful discussions and acknowledge support from the DFG via SFB-631, SFB-TR12, De730/4-3, and the Cluster of Excellence Nanosystems Initiative $\mathrm{Mu}$ nich. O.G. acknowledges support from the NSF under the grant PHY-1314735.
[1] D. A. Wharam, T. J. Thornton, R. Newbury, M. Pepper, H. Ahmed, J. E. F. Frost, D. G. Hasko, D. C. Peacock, D. A. Ritchie, and G. A. C. Jones, Journal of Physics C: Solid State Physics 21, L209 (1988).

[2] B. J. van Wees, H. van Houten, C. W. J. Beenakker, J. G. Williamson, L. P. Kouwenhoven, D. van der Marel, and C. T. Foxon, Phys. Rev. Lett. 60, 848 (1988).

[3] K. J. Thomas, J. T. Nicholls, M. Y. Simmons, M. Pepper, D. R. Mace, and D. A. Ritchie, Phys. Rev. Lett. 77, 135 (1996).

[4] N. J. Appleyard, J. T. Nicholls, M. Pepper, W. R. Tribe, M. Y. Simmons, and D. A. Ritchie, Phys. Rev. B 62, R16275 (2000).

[5] A. Kristensen, H. Bruus, A. E. Hansen, J. B. Jensen, P. E. Lindelof, C. J. Marckmann, J. Nygård, C. B. Sørensen, F. Beuscher, A. Forchel, and M. Michel, Phys. Rev. B 62, 10950 (2000).

[6] S. M. Cronenwett, H. J. Lynch, D. Goldhaber-Gordon, L. P. Kouwenhoven, C. M. Marcus, K. Hirose, N. S. Wingreen, and V. Umansky, Phys. Rev. Lett. 88, 226805 (2002).

[7] L. DiCarlo, Y. Zhang, D. T. McClure, D. J. Reilly, C. M. Marcus, L. N. Pfeiffer, and K. W. West, Phys. Rev. Lett. 97, 036810 (2006).

[8] O. Chiatti, J. T. Nicholls, Y. Y. Proskuryakov, N. Lumpkin, I. Farrer, and D. A. Ritchie, Phys. Rev. Lett. 97, 056601 (2006).

[9] L. W. Smith, A. R. Hamilton, K. J. Thomas, M. Pepper, I. Farrer, J. P. Griffiths, G. A. C. Jones, and D. A. Ritchie, Phys. Rev. Lett. 107, 126801 (2011).

[10] A. P. Micolich, Journal of Physics: Condensed Matter 23, 443201 (2011).

[11] R. Danneau, O. Klochan, W. R. Clarke, L. H. Ho, A. P. Micolich, M. Y. Simmons, A. R. Hamilton, M. Pepper, and D. A. Ritchie, Phys. Rev. Lett. 100, 016403 (2008).

[12] D. J. Reilly, T. M. Buehler, J. L. O'Brien, A. R. Hamilton, A. S. Dzurak, R. G. Clark, B. E. Kane, L. N. Pfeiffer, and K. W. West, Phys. Rev. Lett. 89, 246801 (2002).

[13] K. F. Berggren and I. I. Yakimenko, Phys. Rev. B 66, 085323 (2002).

[14] Y. Meir, K. Hirose, and N. S. Wingreen, Phys. Rev. Lett. 89, 196802 (2002).

[15] C. Sloggett, A. I. Milstein, and O. P. Sushkov, The European Physical Journal B 61, 427 (2008).

[16] A. M. Lunde, A. D. Martino, A. Schulz, R. Egger, and K. Flensberg, New J. Phys. 11, 023031 (2009).

[17] K. Aryanpour and J. E. Han, Phys. Rev. Lett. 102, 056805 (2009).

[18] F. Bauer, J. Heyder, E. Schubert, D. Borowsky, D. Taubert, B. Bruognolo, D. Schuh, W. Wegscheider, J. von Delft, and S. Ludwig, Nature 501, 73 (2013).
[19] M. Kohda, S. Nakamura, Y. Nishihara, K. Kobayashi, T. Ono, J.-i. Ohe, Y. Tokura, T. Mineno, and J. Nitta, Nat Commun 3, 1082 (2012).

[20] J.-H. Hsiao and T.-M. Hong, Phys. Rev. B 82, 115309 (2010).

[21] M. P. Nowak, K. Kolasiński, and B. Szafran, Phys. Rev. B 90, 035301 (2014), arXiv:1401.1707 [cond-mat.meshall].

[22] R. Danneau, W. R. Clarke, O. Klochan, A. P. Micolich, A. R. Hamilton, M. Y. Simmons, M. Pepper, and D. A. Ritchie, Applied Physics Letters 88, 012107 (2006).

[23] F. Nichele, S. Chesi, S. Hennel, A. Wittmann, C. Gerl, W. Wegscheider, D. Loss, T. Ihn, and K. Ensslin, Phys. Rev. Lett. 113, 046801 (2014).

[24] R. Winkler, Spin-Orbit Coupling Effects in TwoDimensional Electron and Hole Systems (Springer Berlin Heidelberg, 2003).

[25] L. Meier, G. Salis, I. Shorubalko, E. Gini, S. Schön, and K. Ensslin, Nature Physics 3, 650 (2007).

[26] C. H. L. Quay, T. L. Hughes, J. A. Sulpizio, L. N. Pfeiffer, K. W. Baldwin, K. W. West, D. Goldhaber-Gordon, and R. de Picciotto, Nature Physics 6, 336 (2010).

[27] M. Büttiker, Phys. Rev. B 41, 7906(R) (1990).

[28] J. E. Birkholz, Spin-orbit interaction in quantum dots and quantum wires of correlated electrons - A way to spintronics?, Ph.D. thesis, Georg-August-Universität zu Göttingen (2008).

[29] J. Nitta, T. Akazaki, H. Takayanagi, and T. Enoki, Phys. Rev. Lett. 78, 1335 (1997).

[30] T. Kita, T. Kakegawa, M. Akabori, and S. Yamada, Solid State Communications 136, 479 (2005).

[31] T. P. Martin, A. Szorkovszky, A. P. Micolich, A. R. Hamilton, C. A. Marlow, R. P. Taylor, H. Linke, and H. Q. Xu, Phys. Rev. B 81, 041303 (2010).

[32] J. C. H. Chen, O. Klochan, A. P. Micolich, A. R. Hamilton, T. P. Martin, L. H. Ho, U. Zülicke, D. Reuter, and A. D. Wieck, New J. Phys. 12, 033043 (2010).

[33] A. R. Hamilton, T. Li, O. P. Sushkov, and R. Winkler, For weakly 1D-confined holes, it can be argued that the dominant SOI term has the same $k \sigma_{y}$ form as used here. Private communication. (2014).

[34] S. Datta, Electronic Transport in Mesoscopic Systems (Cambridge University Press, 1995).

[35] F. Bauer, J. Heyder, and J. von Delft, Phys. Rev. B 89, 045128 (2014).

[36] C. Wetterich, Physics Letters B 301, 90 (1993).

[37] J. E. Birkholz and V. Meden, Phys. Rev. B 79, 085420 (2009).

[38] F. Bauer, 0.7 Anomaly of Quantum Point Contacts: Treatment of Interactions with Functional Renormalization Group., Master's thesis, LMU-München (2008). 
[39] W. Metzner, M. Salmhofer, C. Honerkamp, V. Meden, and K. Schönhammer, Rev. Mod. Phys. 84, 299 (2012).

[40] A. R. Hamilton, K. Hudson, A. P. Micolich, and A. Srinivasan, private communication (2014).

[41] R. M. Lutchyn, J. D. Sau, and S. Das Sarma, Phys. Rev. Lett. 105, 077001 (2010).

[42] Y. Oreg, G. Refael, and F. von Oppen, Phys. Rev. Lett. 105, 177002 (2010).

[43] V. Mourik, K. Zuo, S. M. Frolov, S. R. Plissard, E. P. A. M. Bakkers, and L. P. Kouwenhoven, Science 336, 1003 (2012). 


\section{SUPPLEMENTAL MATERIAL TO "EFFECT OF SPIN-ORBIT INTERACTIONS ON THE 0.7 ANOMALY IN QUANTUM POINT CONTACTS"}

\section{Geometric details of the model}

In our model the 2DES is in the $x y$-plane and the QPC is directed along the $x$-axis (this is the direction of motion of the charge carrier). For the directions of the $\boldsymbol{B}$ and $\boldsymbol{B}_{\mathrm{SOI}}$ fields we impose the following restrictions. To avoid orbital effects we require the magnetic field $\boldsymbol{B}$ to be in the $x y$-plane of the 2DES. We also want to be able to rotate $\boldsymbol{B}$ through any angle $\varphi$ w.r.t. $\boldsymbol{B}_{\mathrm{SOI}}$, which implies that $\boldsymbol{B}_{\mathrm{SOI}}$ also must lie in the $x y$-plane. With the latter condition, the $\boldsymbol{B}_{\mathrm{SOI}}$ field can be either parallel to the direction of motion of the electrons (pure Dresselhaus contribution), or orthogonal to it (pure Rashba contribution), or a combination of the two. But for our mathematical model, the end results depend only on the relative angle $\varphi$ between $\boldsymbol{B}$ and $\boldsymbol{B}_{\mathrm{SOI}}$. This means that we can choose the direction of $\boldsymbol{B}_{\mathrm{SOI}}$ without loss of generality. We choose $\boldsymbol{B}_{\mathrm{SOI}}$ to be parallel to the $y$-axis, c.f. Eq. (2) of the main text.

\section{The spatially resolved LDOS}

In Fig. 1(b1-b3) of the main text we show the LDOS at fixed $\mu$ as a function of $V_{\mathrm{g}}$ on the central site of the QPC. The behavior at the center captures all relevant features. For completeness we include here in Fig. 3 the spatially resolved plots of the $\operatorname{LDOS} \mathcal{A}_{j}^{\sigma}$ for both spin states and the same parameter values as in Fig. 1 of the main text.

\section{Second order fRG}

The functional Renormalization Group (fRG) method is an improved perturbation technique [28, 36-39]. Rather than expanding the Green's function in orders of the coupling and truncating the expansion, fRG introduces a flow parameter $\Lambda$ into the free Green's function $\mathcal{G}_{0}$. At zero temperature we define

$$
\mathcal{G}_{0}(i \omega) \rightarrow \theta(|\omega|-\Lambda) \mathcal{G}_{0}(i \omega) \equiv \mathcal{G}_{0}^{\Lambda}(i \omega)
$$

where $\theta(\omega)$ is the Heaviside step function. At the beginning of the flow, setting $\Lambda=\infty$ yields $\mathcal{G}_{0}^{\Lambda}=0$, which means that the only contribution to the full Green's function comes from the bare vertex. At the end of the flow, $\Lambda=0$ recovers the full model. The technical details of the one-particle irreducible version of the fRG employed in this work are presented in depth in [35]. We use the static approximation at zero temperature, which will be described below in Sec. 3 b. Since [35] does not deal with spin-orbit interactions, no spin-mixing is possible, which introduces additional symmetries into the system.

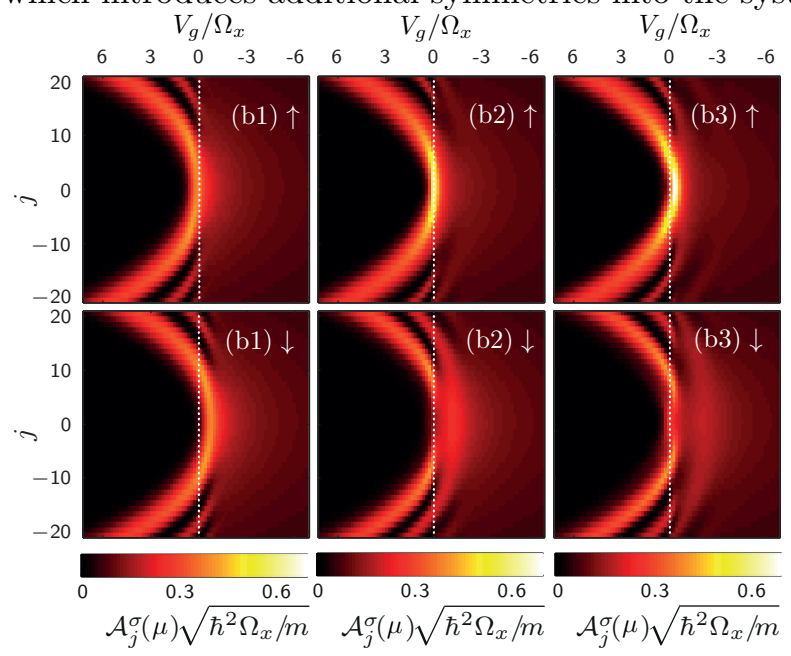

FIG. 3. Spatially resolved plots of the noninteracting LDOS $\mathcal{A}_{j}^{\sigma}$ at fixed $\omega=\mu$, plotted as a function of gate voltage $V_{\mathrm{g}}$ and site index $j$, for $B=0.88 \Omega_{x}$ and for spin $\sigma=\uparrow$ (top row) and $\sigma=\downarrow$ (bottom row). Left column: $R=0.84, \varphi=0$. Middle column: $R=0.84, \varphi=\pi / 2$. Right column: $R=1.26$, $\varphi=\pi / 2$. All results shown are for $N=50$.

In our case these symmetries are no longer present. In this Supplement we focus on the generalizations necessary to account for such spin-mixing terms.

The second-order fRG flow equations in the oneparticle irreducible version and in the static approximation are given by 


$$
\begin{aligned}
\frac{\mathrm{d}}{\mathrm{d} \Lambda} \gamma_{1}^{\Lambda}\left(q_{1}^{\prime}, q_{1}\right) & =\frac{1}{2 \pi} \sum_{\omega= \pm \Lambda} \sum_{q_{2}^{\prime}, q_{2}} \widetilde{\mathcal{G}}_{q_{2}, q_{2}^{\prime}}^{\Lambda}(i \omega) \gamma_{2}^{\Lambda}\left(q_{2}^{\prime}, q_{1}^{\prime} ; q_{2}, q_{1}\right), \\
\frac{\mathrm{d}}{\mathrm{d} \Lambda} \gamma_{2}^{\Lambda} & =\frac{\mathrm{d}}{\mathrm{d} \Lambda}\left(\gamma_{p}^{\Lambda}+\gamma_{x}^{\Lambda}+\gamma_{d}^{\Lambda}\right), \text { where } \\
\frac{\mathrm{d}}{\mathrm{d} \Lambda} \gamma_{p}^{\Lambda}\left(q_{1}^{\prime}, q_{2}^{\prime} ; q_{1}, q_{2}\right) & =\frac{1}{2 \pi} \sum_{\omega= \pm \Lambda} \sum_{q_{3}^{\prime}, q_{3}, q_{4}^{\prime}, q_{4}} \frac{1}{2} \gamma_{2}^{\Lambda}\left(q_{1}^{\prime}, q_{2}^{\prime} ; q_{3}, q_{4}\right) \widetilde{\mathcal{G}}_{q_{3}, q_{3}^{\prime}}^{\Lambda}(i \omega) \widetilde{\mathcal{G}}_{q_{4}, q_{4}^{\prime}}^{\Lambda}(-i \omega) \gamma_{2}^{\Lambda}\left(q_{3}^{\prime}, q_{4}^{\prime} ; q_{1}, q_{2}\right), \\
\frac{\mathrm{d}}{\mathrm{d} \Lambda} \gamma_{x}^{\Lambda}\left(q_{1}^{\prime}, q_{2}^{\prime} ; q_{1}, q_{2}\right) & =\frac{1}{2 \pi} \sum_{\omega= \pm \Lambda} \sum_{q_{3}^{\prime}, q_{3}, q_{4}^{\prime}, q_{4}} \gamma_{2}^{\Lambda}\left(q_{1}^{\prime}, q_{4}^{\prime} ; q_{3}, q_{2}\right) \widetilde{\mathcal{G}}_{q_{3}, q_{3}^{\prime}}^{\Lambda}(i \omega) \widetilde{\mathcal{G}}_{q_{4}, q_{4}^{\prime}}^{\Lambda}(i \omega) \gamma_{2}^{\Lambda}\left(q_{3}^{\prime}, q_{2}^{\prime} ; q_{1}, q_{4}\right), \\
\frac{\mathrm{d}}{\mathrm{d} \Lambda} \gamma_{d}^{\Lambda}\left(q_{1}^{\prime}, q_{2}^{\prime} ; q_{1}, q_{2}\right) & =-\frac{1}{2 \pi} \sum_{\omega= \pm \Lambda} \sum_{q_{3}^{\prime}, q_{3}, q_{4}^{\prime}, q_{4}} \gamma_{2}^{\Lambda}\left(q_{1}^{\prime}, q_{3}^{\prime} ; q_{1}, q_{4}\right) \widetilde{\mathcal{G}}_{q_{4}, q_{4}^{\prime}}^{\Lambda}(i \omega) \widetilde{\mathcal{G}}_{q_{3}, q_{3}^{\prime}}^{\Lambda}(i \omega) \gamma_{2}^{\Lambda}\left(q_{4}^{\prime}, q_{2}^{\prime} ; q_{3}, q_{2}\right),
\end{aligned}
$$

where $-\gamma_{1}^{\Lambda}$ is the self-energy and $\gamma_{2}^{\Lambda}$ is the two-particle irreducible vertex. All higher order vertices $\gamma_{n \geq 3}$ have been set to zero. Here $\widetilde{\mathcal{G}}^{\Lambda}$ is defined as

$$
\widetilde{\mathcal{G}}^{\Lambda}=\left[\mathcal{G}_{0}^{-1}+\gamma_{1}^{\Lambda}\right]^{-1}=\frac{1}{i \omega-H_{0}+\gamma_{1}^{\Lambda}},
$$

where $H_{0}$ is the (known) Hamiltonian of the noninteracting system. The quantum numbers $q_{i}$ encode the spin and spatial degrees of freedom $q \equiv(\sigma, j)$. The flow of $\gamma_{2}^{\Lambda}$ was split into three contributions called the particleparticle channel $(P)$, and the exchange $(X)$ and direct $(D)$ contributions to the particle-hole channel, respectively. This will allow us to simplify the flow equations later.

For a derivation of Eqs. (6-10) see for instance [18, 35, $38]$.

\section{a. Initial condition}

For the numerical treatment we cannot set the initial value of the flow parameter $\Lambda_{\text {init }}$ to infinity, but it is sufficient that it is much larger than all relevant energy scales. We have the following initial condition at $\Lambda_{\text {init }}$ $[18,35,38]$,

$$
\begin{aligned}
\gamma_{2}^{\Lambda_{\mathrm{init}}}\left(q_{1}, q_{2}, q_{3}, q_{4}\right) & =v_{q_{1}, q_{2}, q_{3}, q_{4}}, \\
\gamma_{1}^{\Lambda_{\mathrm{init}}}\left(q_{1}, q_{2}\right) & =-\frac{1}{2} \sum_{q} v_{q_{1}, q, q_{2}, q},
\end{aligned}
$$

where the vertex $v_{q_{1}, q_{2}, q_{3}, q_{4}}$ is site diagonal and at site $j \equiv j_{1}=j_{2}=j_{3}=j_{4}$ is given by

$$
v_{q_{1}, q_{2}, q_{3}, q_{4}}=U_{j} \delta_{\sigma_{1} \bar{\sigma}_{2}}\left(\delta_{\sigma_{1} \sigma_{3}} \delta_{\sigma_{2} \sigma_{4}}-\delta_{\sigma_{1} \sigma_{4}} \delta_{\sigma_{2} \sigma_{3}}\right) .
$$

This means that the spins $q_{1}$ and $q_{2}$, as well as the spins $q_{3}$ and $q_{4}$ must be opposite. This leaves two possibilities: $\sigma_{1}=\sigma_{3}=\bar{\sigma}_{2}=\bar{\sigma}_{4}$ that has positive sign, and $\sigma_{1}=\sigma_{4}=$ $\bar{\sigma}_{2}=\bar{\sigma}_{3}$ that has negative sign. Inserting this into the initial condition for $\gamma_{1}^{\Lambda}$ yields

$$
\gamma_{1}^{\Lambda_{\text {init }}}\left(q^{\prime}, q\right)=-\left(U_{j} / 2\right) \delta_{\sigma^{\prime} \sigma} .
$$

\section{b. Approximations}

We use the following approximations, see [18] and references thereof. Firstly, we neglect the frequency dependence of $\gamma_{2}^{\Lambda}$. This is called the static approximation and is known to give good results at $T=0$ [35]. Given the structure of the flow equation for $\gamma_{2}^{\Lambda}$ above, it is natural to divide the flowing vertex into four parts as follows:

$$
\gamma_{2}^{\Lambda}=v+\gamma_{p}^{\Lambda}+\gamma_{x}^{\Lambda}+\gamma_{d}^{\Lambda} .
$$

Here $v$ is shorthand for the bare vertex, and the flows of $\gamma_{p}^{\Lambda}, \gamma_{x}^{\Lambda}$ and $\gamma_{d}^{\Lambda}$ were given above. The initial value for $\gamma_{2}^{\Lambda}$ is simply the bare vertex. If we insert the bare vertex into the flow equations for the components of $\gamma_{2}^{\Lambda}$ we observe that some of its symmetries remain preserved in the derivative on the left hand side. For instance, for $\gamma_{p}^{\Lambda}$ we see that the first two and the second two site indices must be identical and also that the first and the second pair of spin indices must be opposite, respectively. Similarly, for $\gamma_{x}^{\Lambda}$ we see that the first and fourth site index, as well as the the second and third site index must be equal. For $\gamma_{d}^{\Lambda}$ the first and the third, as well as the second and the fourth site indices are equal. There is no restriction on the any of the spin indices for either $\gamma_{x}^{\Lambda}$ or $\gamma_{d}^{\Lambda}$

The next iteration would break the remaining symmetries, since all three channels contribute to the derivative of $\gamma_{2}^{\Lambda}$ and then back-feed into the differential equations for each channel. If instead we choose to only backfeed each channel into its own differential equation, we can preserve the symmetries described above. This immensely simplifies the treatment of the equations. By doing so, we only neglect contributions of order $v^{3}$ and higher, which justifies their neglect as long as $U_{j}$ is not too large. Altogether we obtain the following contributions:

$$
\begin{aligned}
P_{j i}^{\sigma \bar{\sigma}} & :=\gamma_{p}^{\Lambda}(j \sigma, j \bar{\sigma} ; i \sigma, i \bar{\sigma}) \\
\bar{P}_{j i}^{\sigma \bar{\sigma}} & :=\gamma_{p}^{\Lambda}(j \sigma, j \bar{\sigma} ; i \bar{\sigma}, i \sigma) \\
X_{j i}^{\sigma_{1} \sigma_{2} \sigma_{3} \sigma_{4}}: & :=\gamma_{x}^{\Lambda}\left(j \sigma_{1}, i \sigma_{2} ; i \sigma_{3}, j \sigma_{4}\right) \\
D_{j i}^{\sigma_{1} \sigma_{2} \sigma_{3} \sigma_{4}} & :=\gamma_{d}^{\Lambda}\left(j \sigma_{1}, i \sigma_{2} ; j \sigma_{3}, i \sigma_{4}\right)
\end{aligned}
$$


Note that some elements of a channel can also satisfy the symmetries of another channel. So for instance the diagonal element $P_{j j}^{\sigma \bar{\sigma}}$ has the same symmetries as the diagonal elements $X_{j j}^{\sigma \bar{\sigma} \sigma \bar{\sigma}}$ and $D_{j j}^{\sigma \bar{\sigma} \sigma \bar{\sigma}}$. If we back-feed such elements too we preserve the symmetries in each channel, but obtain a more accurate approximation. Therefore, in each of the three flow equations for the channels of $\gamma_{2}^{\Lambda}$ we replace $\gamma_{2}^{\Lambda}$ on the right hand side by the appropriate channels plus the site diagonal contributions of the other channels that obey the same symmetries. The initial conditions for the three channels follow immediately from (16): $\gamma_{p}^{\Lambda_{\text {init }}}=\gamma_{x}^{\Lambda_{\text {init }}}=\gamma_{d}^{\Lambda_{\text {init }}}=0$. Of course, for the differential equation for $\gamma_{1}^{\Lambda}$ we need the full $\gamma_{2}^{\Lambda}$ which is the sum of all three channels and the bare vertex.

\section{c. Symmetries}

Due to the hermiticity of the Hamiltonian the following relation holds for the Green's function

$$
\mathcal{G}(i \omega)=\mathcal{G}^{\dagger}(-i \omega) \Leftrightarrow \mathcal{G}_{i j}(i \omega)=\mathcal{G}_{j i}^{*}(-i \omega)
$$

We assume that this relation also holds for $\tilde{\mathcal{G}}^{\Lambda}$. If $\gamma_{1}^{\Lambda}$ is hermitian then the assumption is obviously justified. Numerical results indeed confirm that $\gamma_{1}^{\Lambda}$ is hermitian. We also have the following symmetries of $\gamma_{2}$ :

$$
\begin{aligned}
\gamma_{2}\left(q_{1}, q_{2}, q_{3}, q_{4}\right) & =-\gamma_{2}\left(q_{2}, q_{1}, q_{3}, q_{4}\right) \\
& =-\gamma_{2}\left(q_{1}, q_{2}, q_{4}, q_{3}\right) \\
& =\gamma_{2}\left(q_{2}, q_{1}, q_{4}, q_{3}\right)
\end{aligned}
$$

This follows directly from the equation defining the twoparticle vertex, see e.g. [38]. Again we assume that these relations hold also for $\gamma_{2}^{\Lambda}$ and moreover for each of the separate channels. Their consistency with the numerical results will be demonstrated below. Altogether this yields the following symmetry relations for the different channels:

$$
\begin{aligned}
& P_{j i}^{\sigma \bar{\sigma}}=P_{j i}^{\bar{\sigma} \sigma}=-\bar{P}_{j i}^{\sigma \bar{\sigma}} \\
& D_{j i}^{\sigma_{1} \sigma_{2} \sigma_{3} \sigma_{4}}=D_{i j}^{\sigma_{2} \sigma_{1} \sigma_{4} \sigma_{3}}=-X_{j i}^{\sigma_{1} \sigma_{2} \sigma_{4} \sigma_{3}} \\
& X_{j i}^{\sigma_{1} \sigma_{2} \sigma_{3} \sigma_{4}}=X_{i j}^{\sigma_{2} \sigma_{1} \sigma_{4} \sigma_{3}}=-D_{j i}^{\sigma_{1} \sigma_{2} \sigma_{4} \sigma_{3}}
\end{aligned}
$$

We observe that $P^{\uparrow \downarrow}=P^{\downarrow \uparrow}$ and hence the spin indices for $P$ will be dropped from now on, leaving only the site index. The alternative configuration $\bar{P}$ follows completely from $P$ and does not need to be kept track of separately. Same applies to $X$ and $D$ which completely define each other. We choose to work with $D$. There are various symmetries of $D$ but there is no restriction on the spin index. This means that there are $2^{4}=16$ different submatrices corresponding to 16 different spin configurations of $D$. We choose to arrange them as follows

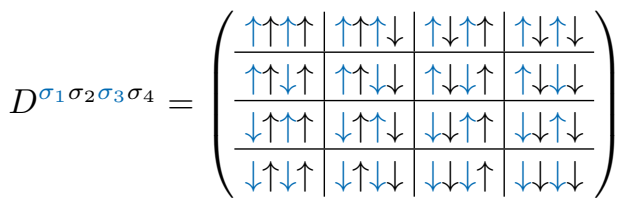

Note that the first and third spin index are fixed along a row and correspondingly the second and fourth index are fixed along one column. This form of the matrix will prove convenient later. From the symmetries of $D$ it follows that this matrix is symmetric. Numerically we also confirm the following relations between the different blocks, schematically

\begin{tabular}{|c|c|c|c|}
\hline$A_{s}$ & $B$ & $B^{*}$ & $C$ \\
\hline$B^{T}$ & $D_{s}$ & $E_{h}$ & $F$ \\
\hline$\left(B^{*}\right)^{T}$ & $E_{h}^{T}$ & $D_{s}^{*}$ & $F^{*}$ \\
\hline$C^{T}$ & $F^{T}$ & $\left(F^{*}\right)^{T}$ & $G_{s}$ \\
\hline
\end{tabular}

where identical symbols denote equal blocks and symmetric (hermitian) submatrices are labeled by the subscript $s(h)$. There are only seven different blocks in total. $\mathrm{Nu}-$ merically we also show that the corner submatrices $A_{s}$, $G_{s}, C$ and $C^{T}$ are real. The other submatrices are complex in general. For a hermitian $\gamma_{1}^{\Lambda}$, the first flow equation implies that $\gamma_{2}^{\Lambda}\left(q_{2}^{\prime}, q_{1}^{\prime} ; q_{2}, q_{1}\right)=\gamma_{2}^{\Lambda *}\left(q_{2}, q_{1} ; q_{2}^{\prime}, q_{1}^{\prime}\right)$. Translated to the separate channels this confirms that $P$ must indeed be hermitian, since $P_{i j}=P_{j i}^{*}$, as well as all the remaining relations between the different submatrices of $D$. 


\section{d. Flow equation for the P-channel}

Restricting $\gamma_{p}^{\Lambda}$ according to the symmetries of the $P$-channel we obtain the following simplified equation for the derivative of $P$ :

$$
\begin{aligned}
& \frac{\mathrm{d}}{\mathrm{d} \Lambda} P_{j i}=\frac{\mathrm{d}}{\mathrm{d} \Lambda} \gamma_{p}^{\Lambda}(j \sigma, j \bar{\sigma} ; i \sigma, i \bar{\sigma}) \\
&=\frac{1}{2 \pi} \sum_{\omega= \pm \Lambda} \sum_{k, l} \frac{1}{2} \cdot\left[\gamma_{2}^{\Lambda}(j \sigma, j \bar{\sigma} ; k \sigma, k \bar{\sigma}) \widetilde{\mathcal{G}}_{k l}^{\Lambda \sigma \sigma}(i \omega) \widetilde{\mathcal{G}}_{k l}^{\Lambda \bar{\sigma} \bar{\sigma}}(-i \omega) \gamma_{2}^{\Lambda}(l \sigma, l \bar{\sigma} ; i \sigma, i \bar{\sigma})\right. \\
&+\gamma_{2}^{\Lambda}(j \sigma, j \bar{\sigma} ; k \bar{\sigma}, k \sigma) \widetilde{\mathcal{G}}_{k l}^{\Lambda \bar{\sigma} \bar{\sigma}}(i \omega) \widetilde{\mathcal{G}}_{k l}^{\Lambda \sigma \sigma}(-i \omega) \gamma_{2}^{\Lambda}(l \bar{\sigma}, l \sigma ; i \sigma, i \bar{\sigma}) \\
&+\gamma_{2}^{\Lambda}(j \sigma, j \bar{\sigma} ; k \sigma, k \bar{\sigma}) \widetilde{\mathcal{G}}_{k l}^{\Lambda \sigma \bar{\sigma}}(i \omega) \widetilde{\mathcal{G}}_{k l}^{\Lambda \bar{\sigma} \sigma}(-i \omega) \gamma_{2}^{\Lambda}(l \bar{\sigma}, l \sigma ; i \sigma, i \bar{\sigma}) \\
&\left.+\gamma_{2}^{\Lambda}(j \sigma, j \bar{\sigma} ; k \bar{\sigma}, k \sigma) \widetilde{\mathcal{G}}_{k l}^{\Lambda \bar{\sigma} \sigma}(i \omega) \widetilde{\mathcal{G}}_{k l}^{\Lambda \sigma \bar{\sigma}}(-i \omega) \gamma_{2}^{\Lambda}(l \sigma, l \bar{\sigma} ; i \sigma, i \bar{\sigma})\right]
\end{aligned}
$$

Note that the first two terms and the last two terms in the sum are equivalent after summation over $\omega$, due to the symmetry relations (23) and (24). We can thus keep one of the terms respectively and cancel the factor of $1 / 2$. With the definitions

$$
\begin{aligned}
& \Pi_{k l}^{p \Lambda(1)}=\frac{1}{2 \pi} \sum_{\omega= \pm \Lambda} \widetilde{\mathcal{G}}_{k l}^{\Lambda \bar{\sigma} \bar{\sigma}}(i \omega) \widetilde{\mathcal{G}}_{k l}^{\Lambda \sigma \sigma}(-i \omega) \\
& \Pi_{k l}^{p \Lambda(2)}=\frac{1}{2 \pi} \sum_{\omega= \pm \Lambda} \widetilde{\mathcal{G}}_{k l}^{\Lambda \bar{\sigma} \sigma}(i \omega) \widetilde{\mathcal{G}}_{k l}^{\Lambda \sigma \bar{\sigma}}(-i \omega)
\end{aligned}
$$

the flow equation can be written more succinctly as

$$
\begin{aligned}
\frac{\mathrm{d}}{\mathrm{d} \Lambda} P_{j i} & =\sum_{k l}\left[\gamma_{2}^{\Lambda}(j \sigma, j \bar{\sigma} ; k \sigma, k \bar{\sigma}) \Pi_{k l}^{p \Lambda(1)} \gamma_{2}^{\Lambda}(l \sigma, l \bar{\sigma} ; i \sigma, i \bar{\sigma})+\gamma_{2}^{\Lambda}(j \sigma, j \bar{\sigma} ; k \sigma, k \bar{\sigma}) \Pi_{k l}^{p \Lambda(2)} \gamma_{2}^{\Lambda}(l \bar{\sigma}, l \sigma ; i \sigma, i \bar{\sigma})\right] \\
& =\sum_{k l} \gamma_{2}^{\Lambda}(j \sigma, j \bar{\sigma} ; k \sigma, k \bar{\sigma})\left[\Pi_{k l}^{p \Lambda(1)}-\Pi_{k l}^{p \Lambda(2)}\right] \gamma_{2}^{\Lambda}(l \sigma, l \bar{\sigma} ; i \sigma, i \bar{\sigma}),
\end{aligned}
$$

where in the last step we used symmetry relation (23). If we now define

$$
\Pi_{k l}^{p \Lambda} \equiv \Pi_{k l}^{p \Lambda(1)}-\Pi_{k l}^{p \Lambda(2)}=\frac{1}{2 \pi} \sum_{\omega= \pm \Lambda}\left[\widetilde{\mathcal{G}}_{k l}^{\Lambda \bar{\sigma} \bar{\sigma}}(i \omega) \widetilde{\mathcal{G}}_{k l}^{\Lambda \sigma \sigma}(-i \omega)-\widetilde{\mathcal{G}}_{k l}^{\Lambda \bar{\sigma} \sigma}(i \omega) \widetilde{\mathcal{G}}_{k l}^{\Lambda \sigma \bar{\sigma}}(-i \omega)\right]
$$

we arrive at

$$
\frac{\mathrm{d}}{\mathrm{d} \Lambda} P_{j i}=\tilde{P}_{j k} \Pi_{k l}^{p \Lambda} \tilde{P}_{l i}
$$

where $\tilde{P}$ equals $P$ plus the diagonal contributions from the other channels which have the same symmetries as $P$. Explicitly we get

$$
\tilde{P}_{j k}=P_{j k}+\delta_{j k}\left(X_{j j}^{\sigma \bar{\sigma} \sigma \bar{\sigma}}+D_{j j}^{\sigma \bar{\sigma} \sigma \bar{\sigma}}+U_{j}\right)=P_{j k}+\delta_{j k}\left(-D_{j j}^{\sigma \bar{\sigma} \bar{\sigma} \sigma}+D_{j j}^{\sigma \bar{\sigma} \sigma \bar{\sigma}}+U_{j}\right)
$$

Note also that the matrix $\Pi_{k l}^{p \Lambda}$ is hermitian, due to the symmetry (21) of the Green's function.

\section{e. Flow equation for the D-channel}

Restricting $\gamma_{d}^{\Lambda}$ according to the symmetries of the $D$-channel we obtain the following simplified equation for the derivative of $D$ :

$$
\begin{aligned}
\frac{\mathrm{d}}{\mathrm{d} \Lambda} D_{j i}^{\sigma_{1} \sigma_{2} \sigma_{3} \sigma_{4}} & =\frac{\mathrm{d}}{\mathrm{d} \Lambda} \gamma_{d}^{\Lambda}\left(j \sigma_{1}, i \sigma_{2} ; j \sigma_{3}, i \sigma_{4}\right) \\
& =-\frac{1}{2 \pi} \sum_{\omega= \pm \Lambda} \sum_{k l} \sum_{\sigma, \sigma^{\prime}, \sigma^{\prime \prime}, \sigma^{\prime \prime \prime}} \gamma_{2}^{\Lambda}\left(j \sigma_{1}, k \sigma ; j \sigma_{3}, k \sigma^{\prime}\right) \widetilde{\mathcal{G}}_{k l}^{\Lambda \sigma^{\prime} \sigma^{\prime \prime}}(i \omega) \widetilde{\mathcal{G}}_{l k}^{\Lambda \sigma^{\prime \prime \prime} \sigma}(i \omega) \gamma_{2}^{\Lambda}\left(l \sigma^{\prime \prime}, i \sigma_{2} ; l \sigma^{\prime \prime \prime}, i \sigma_{4}\right)
\end{aligned}
$$


Observe that the summation goes over the second and fourth index of the first $\gamma_{2}^{\Lambda}$ matrix and over the first and third index of the second $\gamma_{2}^{\Lambda}$ matrix, while the other indices remain fixed. If we want to recast this expression as a matrix multiplication this indeed implies that the first and third spin index should be fixed along a row and the second and fourth index along one column. This justifies the matrix scheme (28). If we arrange the spin configurations according to this scheme we obtain the matrix equation

$$
\frac{\mathrm{d}}{\mathrm{d} \Lambda} D_{j i}^{\sigma_{1} \sigma_{2} \sigma_{3} \sigma_{4}}=\sum_{k l} \sum_{\sigma, \sigma^{\prime}, \sigma^{\prime \prime}, \sigma^{\prime \prime \prime}} \gamma_{2}^{\Lambda}\left(j \sigma_{1}, k \sigma ; j \sigma_{3}, k \sigma^{\prime}\right) \Pi_{k l}^{d \Lambda \sigma \sigma^{\prime \prime} \sigma^{\prime} \sigma^{\prime \prime \prime}} \gamma_{2}^{\Lambda}\left(l \sigma^{\prime \prime}, i \sigma_{2} ; l \sigma^{\prime \prime \prime}, i \sigma_{4}\right)
$$

where

$$
\Pi_{k l}^{d \Lambda \sigma \sigma^{\prime \prime} \sigma^{\prime} \sigma^{\prime \prime \prime}} \equiv-\frac{1}{2 \pi} \sum_{\omega= \pm \Lambda} \widetilde{\mathcal{G}}_{k l}^{\Lambda \sigma^{\prime} \sigma^{\prime \prime}}(i \omega) \widetilde{\mathcal{G}}_{l k}^{\Lambda \sigma^{\prime \prime \prime} \sigma}(i \omega)
$$

Note that the order of the spin indices on $\Pi$ is not the same as on the Green's functions. The symmetries from (28) remain valid. With our approximation we get

$$
\frac{\mathrm{d}}{\mathrm{d} \Lambda} D_{j i}^{\sigma_{1} \sigma_{2} \sigma_{3} \sigma_{4}}=\tilde{D}_{j k}^{\sigma_{1} \sigma \sigma_{3} \sigma^{\prime}} \Pi_{k l}^{d \sigma \sigma^{\prime \prime} \sigma^{\prime} \sigma^{\prime \prime \prime}} \tilde{D}_{l i}^{\sigma^{\prime \prime} \sigma_{2} \sigma^{\prime \prime \prime} \sigma_{4}}
$$

where $\tilde{D}$ equals $D$ plus the diagonal contributions from the other channels which have the same symmetries as $D$. Explicitly we get

$$
\begin{aligned}
\tilde{D}_{j k}^{\sigma_{1} \sigma_{2} \sigma_{3} \sigma_{4}} & =D_{j k}^{\sigma_{1} \sigma_{2} \sigma_{3} \sigma_{4}}+\delta_{j k}\left(X_{j j}^{\sigma_{1} \sigma_{2} \sigma_{3} \sigma_{4}}+\left(P_{j j}+U_{j}\right) \delta_{\sigma_{1} \bar{\sigma}_{2}}\left(\delta_{\sigma_{1} \sigma_{3}} \delta_{\sigma_{2} \sigma_{4}}-\delta_{\sigma_{1} \sigma_{4}} \delta_{\sigma_{2} \sigma_{3}}\right)\right) \\
& =D_{j k}^{\sigma_{1} \sigma_{2} \sigma_{3} \sigma_{4}}+\delta_{j k}\left(-D_{j j}^{\sigma_{1} \sigma_{2} \sigma_{4} \sigma_{3}}+\left(P_{j j}+U_{j}\right) \delta_{\sigma_{1} \bar{\sigma}_{2}}\left(\delta_{\sigma_{1} \sigma_{3}} \delta_{\sigma_{2} \sigma_{4}}-\delta_{\sigma_{1} \sigma_{4}} \delta_{\sigma_{2} \sigma_{3}}\right)\right)
\end{aligned}
$$

Just like $D$ itself, the matrix $\Pi_{k l}^{d \Lambda \sigma \sigma^{\prime \prime} \sigma^{\prime} \sigma^{\prime \prime \prime}}$ is symmetric, however in general not real. The structure of $\Pi_{k l}^{d \Lambda \sigma \sigma^{\prime \prime} \sigma^{\prime} \sigma^{\prime \prime \prime}}$ in terms of its submatrices is the same as for $D$.

\section{f. Flow equation for $\gamma_{1}$}

For the self-energy equation

$$
\frac{\mathrm{d}}{\mathrm{d} \Lambda} \gamma_{1}^{\Lambda}\left(k^{\prime} \sigma^{\prime}, k \sigma\right)=\frac{1}{2 \pi} \sum_{\omega= \pm \Lambda} \sum_{k_{1}, k_{2}, \sigma_{1}, \sigma_{2}} \widetilde{\mathcal{G}}_{k_{2} k_{1}}^{\Lambda \sigma_{2} \sigma_{1}}(i \omega) \gamma_{2}^{\Lambda}\left(k_{1} \sigma_{1}, k^{\prime} \sigma^{\prime} ; k_{2} \sigma_{2}, k \sigma\right)
$$

we need the full $\gamma_{2}^{\Lambda}=v+\gamma_{p}^{\Lambda}+\gamma_{x}^{\Lambda}+\gamma_{d}^{\Lambda}$. We abbreviate

$$
\mathcal{S}_{k_{2} k_{1}}^{\sigma_{2} \sigma_{1}}=\frac{1}{2 \pi} \sum_{\omega= \pm \Lambda} \widetilde{\mathcal{G}}_{k_{2} k_{1}}^{\Lambda \sigma_{2} \sigma_{1}}(i \omega)
$$

Taking into account the symmetry of each channel we obtain

$$
\begin{aligned}
\frac{\mathrm{d}}{\mathrm{d} \Lambda} \gamma_{1}^{\Lambda}\left(k^{\prime} \sigma^{\prime}, k \sigma\right)= & \delta_{\sigma \sigma^{\prime}} \mathcal{S}_{k k^{\prime}}^{\bar{\sigma} \bar{\sigma}}\left(P_{k^{\prime} k}+\delta_{k k^{\prime}} U_{k}\right)-\delta_{\sigma \bar{\sigma}^{\prime}} \mathcal{S}_{k k^{\prime}}^{\bar{\sigma} \sigma}\left(P_{k^{\prime} k}+\delta_{k k^{\prime}} U_{k}\right) \\
& -\sum_{\sigma_{1} \sigma_{2}} \mathcal{S}_{k^{\prime} k}^{\sigma_{2} \sigma_{1}} D_{k k^{\prime}}^{\sigma_{1} \sigma^{\prime} \sigma \sigma_{2}}+\delta_{k k^{\prime}} \sum_{l, \sigma_{1}, \sigma_{2}} \mathcal{S}_{l l}^{\sigma_{2} \sigma_{1}} D_{l k}^{\sigma_{1} \sigma^{\prime} \sigma_{2} \sigma}
\end{aligned}
$$

The first line accounts for the bare vertex and the $P / \bar{P}$-channel, while the second line contains the contribution from the $X$-channel and then the $D$-channel. Note that the $D$-channel only influences the diagonal elements of $\gamma_{1}^{\Lambda}$, due to its symmetry. 